\section{Chrischta Ganz}

\section{Chronobiologie}

Die Chronobiologie erforscht die natürlichen Rhythmen und Prozesse, die das Leben beeinflussen und sich im Zeitverlauf periodisch wiederholen. Im Körperinneren festgelegte Taktgeber laufen mit unterschiedlicher Geschwindigkeit und Periodenlänge nebeneinander her und müssen kontinuierlich abgestimmt werden. Dazu kommen die äusseren Taktgeber, am deutlichsten sichtbar am Wechsel von hell und dunkel. Von diesem Haupttaktgeber sind diverse untergeordnete Rhythmen abhängig.

Der Mensch als rhythmisches Wesen stellt sich seit Urzeiten auf die sich verändernde Natur im Jahreskreis ein (Tag/Nacht, Jahreszeiten, Mondphasen usw.). Ebenso herrschen im Inneren des Menschen diverse Rhythmen wie tageszeitabhängige Hormonausschüttungen (z.B. Melatonin, aber auch Dehydroepiandrosteron, Testosteron usw.), Blutdruck, Herzfrequenz, biochemische Prozesse, der Bedarf an Nahrungszufuhr sowie der weibliche Zyklus, der 7-Jahres-Rhythmus in der Biographiearbeit und viele mehr.

Der circadiane Rhythmus, umgangssprachlich oft die innere Uhr genannt, bezeichnet die Chronobiologie des endogenen Rhythmus während 24 Stunden. Der Begriff circadian wird aus dem lateinischen Wort circa (dt. ungefähr) und dem griechischen Begriff dies (dt. Tag) gebildet und bezeichnet den Rhythmus von 24 Stunden, wie wir ihn als Dauer eines Tages und einer Nacht kennen. Meist wird der Begriff auf Franz Halberg ( ${ }^{\star}$. Juli 1919), den Begründer der Chronobiologie, zurückgeführt.

\title{
Circadiane Rhythmen
}

Fehlt der Lichtimpuls, z.B. bei Blindheit, wird Melatonin zwar ebenso freigesetzt, aber es stellt sich ein Zeitrahmen von 25 anstelle der üblichen 24 Stunden ein [1]. Da sich alle anderen Rhythmen diesem Zeitrahmen anpassen, können daraus Schwierigkeiten für die Betroffenen entstehen, denn die 25 Stunden lassen sich nicht mit dem normalen Tagesablauf vereinbaren.

Bekannt und weit verbreitet sind die Probleme, wenn sich der Mensch innert kurzer Zeit auf neue Tagesabläufe einstellen muss; typischerweise bei Schichtarbeit oder Interkontinentalflügen. Auch bei der halbjährlichen Umstellung auf Sommer- bzw. Winterzeit brauchen manche Menschen (und Tiere) einige Tage, bis sie ihren Rhythmus wieder gefunden haben.

In der «Lebensordnung», die seit jeher wichtiger Bestandteil der ganzheitlichen Prävention und Therapie ist, findet sich ebenso die Betonung von Rhythmus im Leben. Das Einhalten von Rhythmen (insbesondere Schlaf-Wach-Rhythmus und regelmässige Essenszeiten) ist heute oft nicht einfach, lohnt sich jedoch, wie der Alltag in der Praxis zeigt. Da Licht die Hauptrolle für den circadianen Rhythmus spielt, kann Licht auch entsprechend therapeutisch genutzt werden. Die Lichttherapie, früher in den Höhenkliniken als sogenannte Lichtbäder verordnet, wird heute meist mit Lichttherapielampen oder «lichtbringenden» Pflanzen wie beispielsweise Johanniskraut (Hypericum perforatum) umgesetzt. Dass auch regelmässige Aufenthalte im Freien einem Lichtdefizit entgegenwirken (und sich nicht auf reine Vitamin-DZufuhr reduzieren lassen), sei hier noch einmal betont.

\section{Die innere Uhr}

Die Frage des richtigen Zeitpunkts zeigt sich schon in der griechischen Mythologie, wo neben Chronos, dem griechischen Gott der Zeit, auch Kairos bekannt ist (wenn er auch eine untergeordnete Rolle spielt). Chronos steht für den «Zeitabschnitt», Kairos für «den richtigen Zeitpunkt», im weiteren Sinne auch für eine besondere Chance und Gelegenheit.

Auch therapeutische Massnahmen lassen sich im Einklang mit der inneren Uhr zum richtigen Zeitpunkt umsetzen. Es ist beispielsweise sinnvoll, bei der medikamentösen Therapie darauf zu achten, zu welchem Zeitpunkt Medikamente eingenommen werden, da sie zu bestimmten Tageszeiten besser aufgenommen werden als zu anderen. So ist die Wirksamkeit oral verabreichter Medikamente einerseits von der Aktivität des Magens abhängig. Andererseits wird sie von der Durchblutung der Verdauungsorgane beeinflusst, die in der Nacht und in den frühen Morgenstunden deutlich höher ist als am Nachmittag [1]. Die Chronopharmakologie trägt diesen Aspekten Rechnung und beschäftigt sich mit der Herstellung chronobiologisch sinnvoller Medikamente und Präparate.

Die für den Menschen wichtigen Rhythmen beschränken sich keineswegs auf die 24 Stunden des Tages, sondern betreffen das ganze Leben. Im energetischen Sinne sind auch Geburt und Tod als Wandlungsaspekte des Lebens und Teil der natürlichen Rhythmen zu betrachten.

Sicherlich ist das Licht aber der wichtigste und universellste Taktgeber für (fast) alle Lebewesen. Die lichtempfindlichen Pigmente des Organismus (bei Wirbeltieren in der Retina,

\section{KARGER}

Fax +4976145207 14
(๑) 2013 S. Karger GmbH, Freiburg 
bei Insekten und Pflanzen in anderen Zellen) reagieren auf Licht. Bei Pflanzen unterscheidet man neben Chlorophyll die Phytochrome (für rotes Licht) und die Cryptochrome (für blaues Licht). Das Phänomen, dass Pflanzen sich der Sonne zuwenden (Phototropismus), wird den Phototropinen, einer weiteren Klasse der lichtempfindlichen Pigmente, zugesprochen [1]. Man erinnere sich an dieser Stelle an den französischen Namen für die Sonnenblume: Tournesol. Die Ausrichtung des Blütenkopfes zur Sonne hin ist bei der Sonnenblume besonders gut zu beobachten.

Ende des 20. Jahrhunderts wurden bei vielen Säugetieren sogenannte Clock-Gene (Uhrengene) lokalisiert. Diese auch beim Menschen nachgewiesenen Clock-Gene werden durch die Lichtmenge und die Lichtintensität beeinflusst. So ist es gut nachvollziehbar, dass Rhythmus direkt mit der Lichtwahrnehmung gekoppelt ist. Der Nucleus suprachiasmaticus (SCN) ist direkt hinter dem Chiasma opticum gelegen, ist etwa so gross wie ein Reiskorn und gilt als Masterorgan der chronobiologischen Rhythmen. Je nach Tageszeitpunkt und Lichtintensität schüttet er Hormone aus, die direkt auf den Hypothalamus einwirken [2]. Organisiert wird die Biorhythmik aber neben dem SCN auch durch die Epiphyse und durch den Sehfarbstoff Melanopsin. Die Epiphyse ist für die Melatonin-Ausschüttung zuständig. Der Sehfarbstoff Melanopsin befindet sich in der Retina und reagiert nicht auf Farben oder Formen, sondern leitet ausschliesslich die Information «hell» oder «dunkel» an den SCN weiter. Uhrengene konnten jedoch nicht nur im Gehirn und im Auge, sondern auch in der Haut, in den Schleimhäuten, im Herz sowie in der Lunge, Bauchspeicheldrüse und Leber nachgewiesen werden [2].

Aus dem Wissen der Chronobiologie heraus lassen sich auch die beiden Menschentypen erklären, die im Volksmund die Eulen und die Lerchen genannt werden. $\mathrm{Ob}$ der einzelne

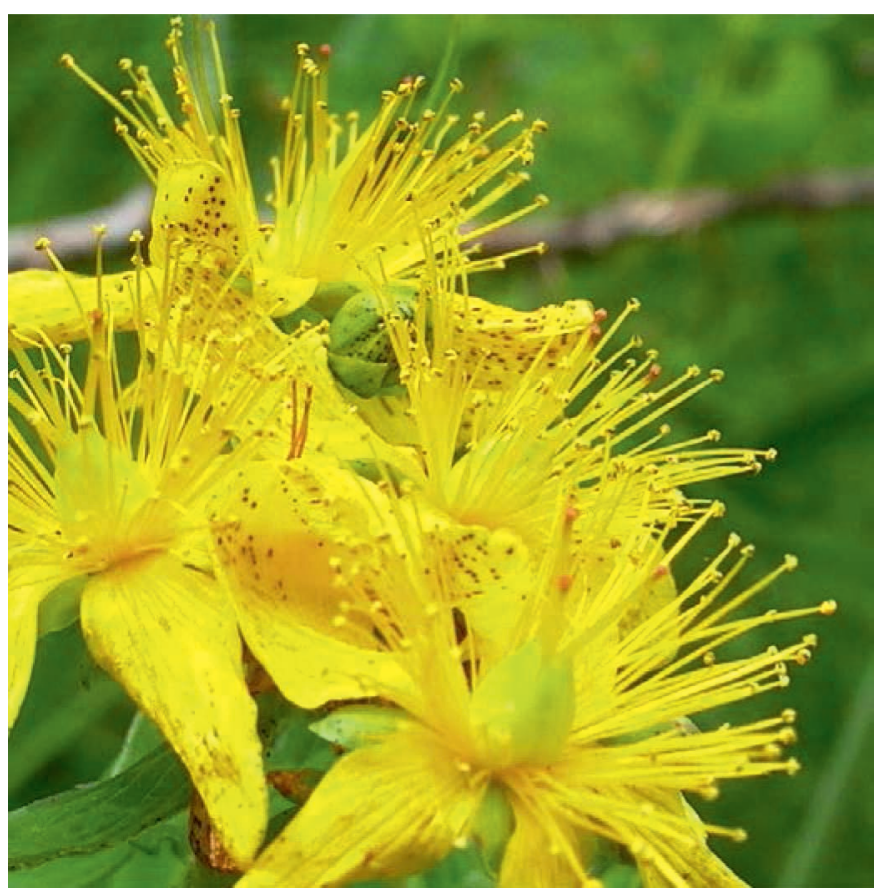

Abb. 1. Johanniskraut als Lichtbringer.

Mensch nun gerne spät ins Bett geht und morgens länger schläft oder gerne früh aus den Federn springt, aber auch früh schlafen geht, ist einerseits eine Typenfrage, andererseits aber auch vom Lebensabschnitt (in der Pubertät ist der Anteil an Eulen deutlich höher) und von sozialen Aspekten (Arbeitszeiten, familiäre Einbindung usw.) abhängig [1].

Typische Symptome bei einer durcheinander geratenen inneren $\mathrm{Uhr}$ sind Schlaf- und Essstörungen, Nervosität, Energielosigkeit, Depressionen, Konzentrationsschwäche und viele mehr. Ausserdem unterstützt ein Leben nach der inneren Uhr jede präventive oder therapeutische Massnahme im Sinne der Ordnungstherapie. Die natürlichen Rhythmen zu respektieren ist eine effektive Möglichkeit, körperliches und psychisches Gleichgewicht zu finden - ein Gleichgewicht, das niemals ein Zustand, sondern immer dynamisch ist [3].

\section{Biorhythmus des Tages}

Dass der Mensch stark auf Rhythmus reagiert, findet auch Ausdruck in den sogenannten Organuhren, die in den meisten naturheilkundlichen Medizinsystemen bekannt sind, allen voran in der Traditionellen Chinesischen Medizin (TCM) [3-5]. Die Organuhr gibt für die einzelnen Organe die Phasen ihrer maximalen Aktivität und ihre Ruhe- bzw. Regenerationszeiten während des Tages an. Die energetische Aktivität durchläuft im Laufe von 24 Stunden jeweils eine Hochphase und 12 Stunden später eine Tiefphase.

\section{Die Organuhr}

Beispiele aus dem Praxisalltag (Tab. 1):

- Erwachen zur Leberzeit: Wenn ein Patient regelmässig zur Leberzeit (zwischen 1 und $3 \mathrm{Uhr}$ in der Nacht) erwacht, verbessert sich der Schlaf bei Gabe eines Lebermittels.

- Pflanzenheilkunde: Sehr bekannt als «Lichtbringer» ist das Johanniskraut (H. perforatum) (Abb. 1). In der Pflanzenheilkunde kennt man ausserdem sogenannte «Rhythmusgeber-Pflanzen», beispielsweise die Brennnessel (Urtica dioica) 
Tab. 1. Die Organuhr.

\begin{tabular}{lll}
\hline Organ & Hochphase & Tiefphase \\
\hline Magen & $07-09$ & $19-21$ \\
Milz und Pankreas & $09-11$ & $21-23$ \\
Herz & $11-13$ & $23-01$ \\
Dünndarm & $13-15$ & $01-03$ \\
Harnblase & $15-17$ & $03-05$ \\
Nieren & $17-19$ & $05-07$ \\
Kreislauf & $19-21$ & $07-09$ \\
Dreifacher Erwärmer & $21-23$ & $09-11$ \\
Gallenblase & $23-01$ & $11-13$ \\
Leber & $01-03$ & $13-15$ \\
Lunge & $03-05$ & $15-17$ \\
Dickdarm & $05-07$ & $17-19$ \\
\hline
\end{tabular}

in der Anthroposophie und den Frauenmantel (Alchemilla xanthochlora) in der Frauenheilkunde.

- Spagyrik: Als Rhythmustherapie bei generellem Rhythmusverlust, aber auch bei Energiemangel und Schlafstörungen haben sich Gaben von spagyrisch aufbereitetem Gold (Aurum spag.) am Morgen in Kombination von spagyrisch aufbereitetem Silber (Argentum spag.) in den Abendstunden bewährt.

\section{Literatur}

1 Fauteck JD: Chronobiologie. Leben mit der inneren Uhr. OM Z Orthomol Med 2006;3.

2 Jahn C: Chronobiologische Störungen. Naturheilpraxis 3/2011.

3 Raimann C, Ganz C, Garvelmann F, Bertschi-Stahl H-D, Fehr-Streule R: Grundlagen der Traditionellen Europäischen Naturheilkunde. Schiedlberg, Bacopa, 2012.

4 Bierbach E: Naturheilpraxis heute. München, Urban und Fischer, 2009.

5 Paungger J, Poppe T: Vom richtigen Zeitpunkt. München, Heinrich Hugendubel, 1991. 